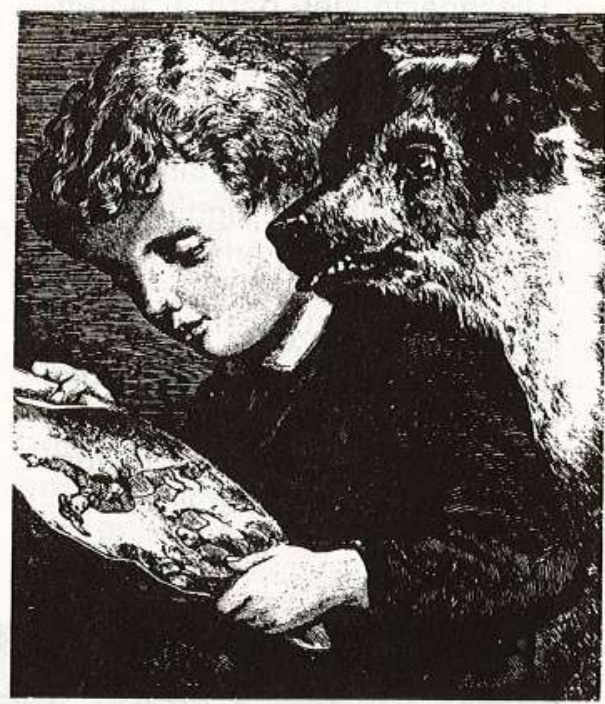

\title{
MODELO DE VALORACIÓN E INTERVENCIÓN EN PROBLEMAS DE APRENDIZAJE
}

Juan de Dios Arias S. Profesor Asociado Pedagógica Nacional, 2000

Nociones preliminares.

Hacia un concepto de problema de aprendizaje

La expresión "problema de aprendizaje" (PA) hace relación a la dificultad de un estudiante para aprehender los contenidos curriculares propios del grado escolar al cual asiste y en los que finalmente presenta bajo rendimiento académico ${ }^{1}$. Un intento

1 El rendimientoacadémico expresado en calificaciones que se asumen describen inicial de taxonomía de los problemas de aprendizaje, conduce al reconocimiento de dos categorias: La primera hace referencia a los problemas de aprendizaje determinados por los ambientes actuales en que vive el estudiante, y no son atribuibles a daños en el sistema nervioso, limitación sensorial severa, problemas físicos o de salud, retardo mental, autismo o a perturba-

los logros de un estudiante respecto de los contenidos curriculares. ción emocional (PA del ambiente presente (PAA)); la segunda, es la de los PA causados por discapacidades para el aprendizaje $(D A)^{2}$, debidas a daño en el

2 La expresión discapacidad para el aprendizaje (DA), corresponde a learning disabilities del inglés. El término "discapacidad" fue aceptado hace más de diez años por la Real Academia Española de la Lengua. Implica falta de habilidad o capacidad de un individuo para algo. Hace referencia a factores intrínsecos. No es correcto traducir disability por dificultad. Asi, la instrucción inadecuada causa dificultad para el aprendizaje pero no una discapacidad para el aprendizaje. 
sistema nervioso. Su definición se describirá en detalle más adelante, hoy, dichas discapacidades son tratadas por la rama especializada de la educación especial, que lleva su nombre. Son estas últimas de naturaleza esencialmente intrínseca al estudiante. Sin embargo, estas dos categorías en algunas ocasiones se traslapan e impiden diferenciar con precisión la etiología de un problema de aprendizaje determinado. Por ejemplo, un problema en el área de la lectoescritura puede originar perturbación emocional o esta última, en otra situación, ser la que origina un problema con la lectoescritura, o también, el problema de aprendizaje de la lectura de un niño puede deberse a "instrucción" deficiente (ambiente extrínseco presente) o a "discapacidad para el aprendizaje" (naturaleza intrínseca).

Problemas de Originados por factores aprendizaje intrínsecos al estudiante. Discapacidades para el Aprendizaje (DA). Son área de la Educación Especial.

\section{DISCAPACIDADES PARAEL APRENDIZAJE (DA): DEFINICIÓN}

Consideremos ahora, la versión de 1989 de la definición, de discapacidad para el aprendizaje, propuesta por la NJCLD ${ }^{3}$. En

3 NJCLD: National Joint Committee on Learning Disabilities. esta, se modifica la de 1981 agregando nuevos contenidos y cambiando algunas palabras.

"Discapacidades para el aprendizaje (Learning disabilities) es una denominación que se refiere a un grupo heterogéneo de desórdenes que se manifiestan como dificultades significativas en la adquisición y empleo de las habilidades del escuchar, hablar, leer, escribir, razonar y la de las matemáticas. Estos desórdenes son intrínsecos al individuo, se presume son debidos a disfunciones del sistema nervioso cen- tral y pueden persistir durante toda la vida. Problemas en las conductas autoregulatorias, la percepción social, y la interacción social pueden coexistir con las discapacidades para el aprendizaje pero no constituir por si mismas una discapacidad para el aprendizaje. Aurique las discapacidades para el aprendizaje pueden ocurrir concomitantemente con otras discapacidades (por ejemplo, deterioro sensorial, retardo mental, perturbación emocional seria), o con influencias extrínsecas (tales como las diferencias culturales, instrucción

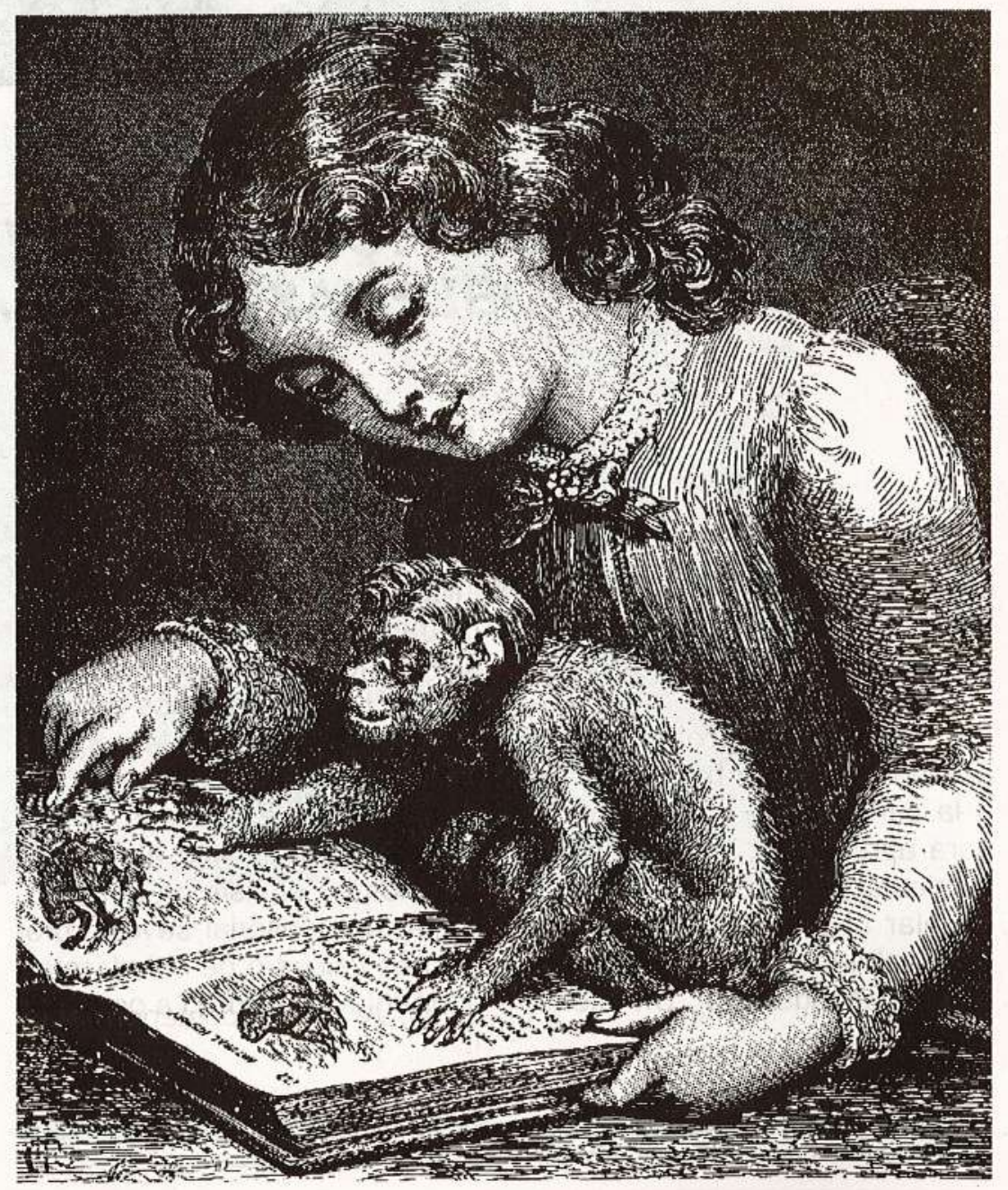


insuficiente e inapropiada), no son el resultado de dichas condiciones e influencias."

En resumen, un niño tendrá una discapacidad para el aprendizaje si:

1. Si su rendimiento no está acorde con lo esperado para su edad y sus niveles de habilidad en una o más de las áreas nombradas a continuación, en el parágrafo a) del numeral 2.

Se supone que al niño se le proveen experiencias de aprendizaje apropiadas a su edad $y$ niveles de habilidad.

2. Existe una severa discrepancia entre el rendimiento y la habilidad intelectual en una o más de las siguientes áreas:

\section{a) i) Expresión oral.}

ii) Comprensión oral.

iii) Expresión escrita.

iv) Destreza básica de lectura.

v) Comprensión de lectura.

vi) Cálculo matemático.

vii) Razonamiento matemático.

b) A un niño no se le identificará como con discapacidad para el aprendizaje si la discrepancia severa entre su habilidad y el rendimiento en las áreas mencionadas resulta de:

1- Limitación visual, auditiva o motora.

II- Retardo Mental.

III- Perturbación Emocional.

IV- Factores ambientales, culturales o económicos.

\section{PROBLEMAS DE APRENDIZAJE ORIGINADOS EN EL AMBIENTE PRESENTE (PAA)}

Corresponden al presenten acápite, la multitud de problemáticas que a diario deterioran el rendimiento académico, de los estudiantes del sistema educativo regular, no afectados por limitaciones físicas, mentales o sensoriales que los hagan sujetos de la educación especial. Es claro que dichas problemáticas, no constituyen el problema de aprendizaje sino que dada su presencia, afectan el rendimiento académico de los estudiantes. De nuevo, recuérdese la descripción de PA hecha antes al considerarlo como un evento. Los problemas de aprendizaje originados en el ambiente presente (PAA) afectan al estudiante "normal", típico de cualquier sistema educativo. Por ende, no se consideran del tipo PAA cuando son causados por limitaciones, severas del estudiante, visuales, auditivas, físicas, motoras, de carácter neurológico o de salud.

Tampoco se incluyen en esta categoría los originados en perturbación emocional seria, el autismo o el retardo mental. Sin embargo, los PAA pueden presentarse adicionalmente en los niños con las limitaciones y/o perturbaciones descritas en las dos últimas frases. En otras palabras, se está afirmando que los PAA pueden ocurrir en niños con necesidades educativas especiales. Al hablar posteriormente de los dominios $u$ áreas que deben ser examinadas, al intervenir en el problema de aprendiza- je de un estudiante, mencionaremos más en detalle factores ambientales de la institución educativa, la familia, la comunidad local, etc., ambientes con los cuales interactúa el estudiante.

\section{VALORACIÓN ${ }^{4}$ DEL NIÑO CON PROBLEMAS DE APRENDIZAJE}

Los cambios conceptuales que en los últimos años ha venido experimentando la educación, se reflejan en su terminología y en la forma de abordar muchas de sus actividades. En el caso de la valoración o estudio del niño con posibles problemas de aprendizaje, se deja de lado la terminología médica que a través de la psicología llegó a la educación. Así, palabras como diagnóstico, pronóstico, test, terapia, empiezan a desaparecer dentro del vocabulario de los educadores, incluidos en éstos los psicopedagogos o psicólogos educativos en sus diferentes especialidades: orientadores, educadores especiales y expertos en PA., etc. Tras de dichas palabras, hace presencia el "modelo médico" que en la educación asumió al niño con PA o necesidades educativas especiales como a un enfermo que debe ser diagnosticado, sometido a una terapia... Por lo general, el diagnóstico apoyado en pruebas psicológicas, se daba en forma de etiqueta: disléxico, retardado mental. Al igual que en la medicina, un vademécum de "remedios o tera-

\footnotetext{
4 Valoración por assessment del inglés. No es sinónimo de evaluation que si se traduce por evaluación.
} 
pias" eran prescritas. Hoy, la educación reconoce cómo muchos de los PA, no "están" en el niño, cómo los problemas de lectura son tan diferentes de niño a niño cómo lo son ellos entre sí, por ende, términos genéricos como el de dislexia, no corresponden a la problemática particular de un niño determinado. Tan inadecuado proceder generó, entre otras consecuencias, el movimiento antietiquetas, que en sus pocos años ha logrado evitar que se continúe aplicando a los niños etiquetas denigrantes y dañinas como las de retardado, sordo, disléxico,... De otra parte, términos como "evaluación" quedan cada vez más relegados a procesos que tengan que ver con mediciones y con pruebas psicológicas.

Al hablar de valoración, estamos estudiando las características de un niño, que son necesarias para poder establecer un programa específico que aborde sus necesidades educativas. Ello implica reunir información mediante técnicas formales e informales aplicadas en los diferentes contextos en los cuales transcurre la vida del niño que se valora.

Los procedimientos incorporados en las etapas que se describen a continuación, permitirán diferenciar si el PA entre manos corresponde a una discapacidad para el aprendizaje (DA), o si es del tipo que se ha llamado problema de aprendizaje de base ambiental (PAA).

Las etapas 1 y 2 corresponden a la llamada detección por otros autores. Según el modelo presente, al finalizar la etapa 2 , la gran mayoría de problemas de tipo PAA, quedan resueltos, a la vez que se inician valoraciones formales y más detalladas de los problemas que no han sido resueltos y que dadas sus características, pasarán en su gran mayoría, una vez terminada la etapa 3 , a clasificarse como de tipo DA, objeto de la educación especial. Como se ve, en la etapa 1 , se elabora una valoración (o evaluación) informal, con instrumentos y procedimientos propios de docentes; además, un enfoque ecológico ${ }^{5}$ debe siempre señalar las situaciones en que se van a recoger los datos que describirán la problemática y fortalezas del estudiante, así como a los recolectores y proveedores de dichos datos, en la etapa 2 se diseñan y aplican intervenciones ad hoc las cuales, la más de las veces, logran que el niño resuelva su situación problemática, si ello ocurre, es porque el PA, correspondía a la forma PAA. En la etapa 3, se habla de evaluación formal por profesionales pertinentes al caso y se espera sólo pasen a ella estudiantes que se sospecha pueden terminar descritos como con DA. En ésta, intervendrán, además de los miembros del equipo que participó hasta el fin de la etapa 2 , profesionales cuyos conocimientos sean los señalados para intervenir, ante las características dominantes del PA específico que se esté tratando, v.gr: fonoaudiólogo, terapeuta físico,...

5 DOCKRELL, J. y MC Shane, J. (1992) Children's Learning Difficulties. A Cognitive Approach. Oxford, UK: Blackwell Pub.

\section{Etapa 1. Descripción informal de la problemática y fortalezas del estudiante ${ }^{6}$}

En la lectura sobre Técnicas de Valoración, preparada para el Sexto Congreso de Orientación Educativa, se describen los conceptos de valoración informal y valoración formal, además de sus aplicaciones.

Un equipo ad hoc conformado por el maestro de aula, los padres del estudiante y el estudiante mismo, bajo la dirección del orientador escolar, o el educador especial o el psicopedagogo, cuando la institución escolar cuenta con sus servicios, es suficiente para iniciar el estudio de la problemática presentada por un estudiante con el fin de describirla.

La descripción que el equipo elabore, debe incorporar información sobre las fortalezas del estudiante además de su problemática, vista en función de los contextos diferentes (colegio, hogar, vecindario,...) en que a diario se desempeña y en los dominios que para el problema de aprendizaje entre manos, se considere pertinente. La citada lectura, sobre Técnicas de Valoración, reseña a manera de orientación para los especialistas en PA los principales dominios a conside-

\footnotetext{
- Muchas de las ideas incorporadas en las siguientes etapas han sido tomadas y adaptadas de:

NJCLD. (1997) Operationalizing the NJCLD Definition of Learning Disabilities for Ongoing Assessment in Schools. Autor. Internet: http.//www.idonline.org/njcld/ operationalizing.html
} 


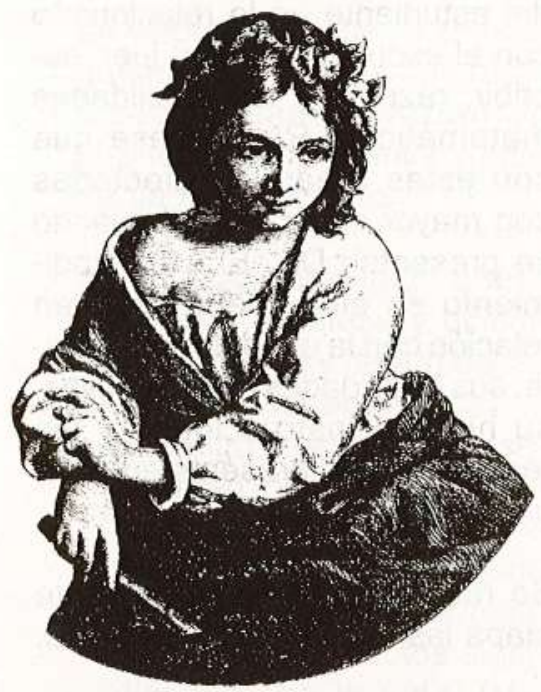

rar. De otra parte, las responsabilidades del equipo son:

A. Identificar personas que tengan información sobre el estudiante, su problemática en general, características del hogar, del vecindario, de sus compañeros de barrio, etc. y entrevistarlas (además de aportar la que ellos mismos conocen; es cuando los llamados enfoques ecológicos muestran su potencia investigativa). Tener presente que el bajo rendimiento académico de un estudiante puede estar relacionado con lo que afecta al niño en el hogar, vecindario, grupo de amigos,... Siempre ha existido la tendencia a creer que los PA de un niño tienen siempre su origen dentro de él. Los ambientes que rodean al niño deben estudiarse en relación con los PA de éste, en unos casos originan PA, en otros es necesario modificarlos para intervenir adecuadamente en la solución de un PA determinado.
B. Reunir y analizar información acerca del aula y las ejecuciones del estudiante en las áreas de interés relacionadas con la problemática:

a) Observación y descripción del aula y las actuaciones del estudiante.

b) Precisar exactamente las áreas académicas en las cuales hay PA.

c) Identificar la problemática emocional asociada a la situación.

d) Precisar exactamente comportamientos inadecuados del estudiante en diferentes sitios.

e) Revisar los registros escolares (contenidos curriculares que se estudian), la historia académica del estudiante (calificaciones, problemas, preferencias, asistencia, participación,...)

f) Revisar cuadernos de tareas del estudiante, trabajos de arte, creación, etc. Comparar lo anterior con las realizaciones de otros estudiantes promedio del aula.

C. Plantear explicaciones plausibles respecto de la problemática del estudiante. Tener en cuenta:

a) Instrucción insuficiente o inadecuada.

b) Metacognición

c) Otros factores importantes que no constituyen por sí mismos discapacidades para el aprendizaje, a saber:

- Comportamientos autoregulatorios (V.g., la atención, la motivación y la impulsividad no constituyen por si mismas DA, aunque la autorregulación de conductas para la ejecución de tareas relacionadas con el lenguaje, lo académico o lo educativo, puede ser una parte integral de alguna discapacidad para el aprendizaje.

- Percepción social (V.g., los juicios sociales inapropiados, no son una DA, pero pueden manifestar consecuencias secundarias de alguna)

- Interacción social (V.g., los problemas relacionados con los compañeros, no constituyen por sí mismos una DA, pero en algunos casos se asocian a ella.)

d) Otras discapacidades tales como)

- Limitaciones sensoriales (descartar problemas de visión y audición)

- Retardo Mental.

- Perturbación emocional severa.

Cuando las anteriores están presentes, la situación debe ser vista desde el ángulo de la educación especial para la discapacidad detectada.

e) Diferencias culturales y lingüísticas.

\section{Etapa 2. Propuesta de interven- ción inicial e implementación de la misma}

Una vez precisada la descripción objeto de la etapa anterior, el equipo procederá a diseñar y poner en marcha acciones que apoyen al estudiante en la superación de su problemática. Para ello: 
A. Elaborará y debatirá diferentes propuestas de solución. (intervenciones, adaptaciones, modificaciones ambientales, remisión a especialistas para valoraciones $\mathrm{u}$ apoyos)

B. Recomendará e implementará las intervenciones u adaptaciones que se determinó satisfacen las necesidades de los estudiantes. (V.g., si el equipo identificó un problema de conciencia fonémica que puede colocar al estudiante en situación de riesgo con su aprendizaje posterior en lectura, se debe entonces intervenir para evitar que fracase.)

C. Vigilará las intervenciones o adaptaciones y hará los ajustes que se vayan necesitando.

D. Evaluará la efectividad de las intervenciones $\mathrm{u}$ adaptaciones.

E. Tomará decisiones según corresponda a:

a) Si las acciones implementadas resultan efectivas para resolver la problemática del estudiante, se continúa con ellas hasta donde se considere necesario. En este caso, se puede con toda seguridad afirmar que el problema resuelto corresponde a la clase PAA.

b) $\mathrm{Si}$ el proceso de solución del problema no resulta efectivo, se examina para verificar su adecuación a la situación; si dicho proceso es descartado, se ensaya otro que se considere adecuado o se decide que es necesaria una valoración más detallada, elaborada con la intervención de otros profesionales cuyo saber sea pertinente a la situación problemática que se maneja.

Los PA que persisten, pueden ser DA y es en la tercera etapa cuando se determina su calidad y existencia. En la cuarta etapa, se procede a diseñar un PEP para el niño detectado con una DA.

\section{Etapa 3. Identificación del es- tudiante como afectado por DA.}

En esta etapa, además de la información obtenida en las etapas 1 y 2 , es necesario efectuar valoraciones informales adicionales, además de las formales, que otros profesionales ahora invitados a conformar un equipo multidisciplinario, deben realizar. La descripción de las características del aprendiz, valoradas ahora en más detalle y con mayor precisión, permitirán con mayor probabilidad determinar que está afectado por una DA. Con toda claridad debe quedar establecida la naturaleza de los problemas de aprendizaje, su severidad y persistencia. Destacar si la ejecución del estudiante en las áreas problemas, es inesperadamente baja cuando se las compara con la ejecución en otras áreas. En fin, precisar si el problema de aprendizaje es realmente el resultado de una DA y no son posibles otras explicaciones al respecto.

El equipo multidisciplinario de profesionales relacionados con la problemática, debe ahora precisar las fortalezas y debilidades del estudiante en lo relacionado con el escuchar, hablar, leer, escribir, razonar y las habilidades matemáticas. Recuérdese que son estas áreas las afectadas con mayor especificidad cuando se presentan DA. El bajo rendimiento en ellas, debe estar en relación con la edad del estudiante, sus habilidades cognoscitivas, su historia instruccional, y los resultados que presente en otras áreas académicas.

Se recomiendan en la presente etapa las siguientes actividades:

A. Identificar aspectos que interfieran con el escuchar, hablar, leer, escribir, razonar o con las habilidades matemáticas cuando se proveen al estudiante oportunidades educativas para superar la situación problemática. Para ello se sugiere:

a) Repasar la información existente respecto del problema (resultados de entrevistas, observaciones,...)

b) Verificar que los datos obtenidos sean confiables y válidos.

c) Ejecutar valoraciones que correspondan a contextos definidos.

d) Analizar la forma de las tareas y de los errores que se presentan.

e) Precisar los conocimientos previos del estudiante.

f) Proveer instrucción de apoyo fundamentada en los saberes previos del estudiante.

g) Programar actividades "controladas" de enseñanza con el fin de observar sus resultados. 
La valoración debe reconocer que las DA, al igual que otras discapacidades, varían en sus formas de manifestarse, de tal manera que:

a) Abarcan diferentes patrones de fortaleza y dificultad a través y dentro de las áreas pertinentes;

b) El rango de expresión y severidad de las $D A$, varía con las demandas de las etapas de la vida y sus diferentes contextos, aunque todas tienen efectos significativos en la vida. (Ver Cap. I)

c) Las DA pueden presentarse de manera diferente en contextos también diferentes, ya sean académicos o no.

Ningún instrumento, lista de características del niño, etc., ha demostrado suficiencia para determinar que un niño padece de DA. En realidad, sólo la práctica, destreza y cualificación de los examinadores, quienes utilizan los instrumentos y técnicas de valoración, es lo que garantiza la corrección de los resultados relacionados con la existencia o no de DA en un niño determinado.

Valorar en detalle las áreas señaladas en la definición de DA, es lo que resta. Ellas son: Las llamadas artes del lenguaje: escuchar, hablar, leer y escribir; el razonamiento y las habilidades matemáticas.

En las etapas de la vida más avanzadas, las dificultades se pueden presentar como pro- blemas del pensamiento abstracto cuantitativo.

B. Se debe también analizar las diferencias de procesamiento en las áreas afectadas. Ver dificultades que involucren memoria, atención, secuencias temporales, planeamiento y coordinación motora, flexibilidad en el pensar, razonar y organizar.

C. Considérense evidencias cuantitativas y cualitativas intraindividuales dentro de las debilidades y fortalezas del aprendiz. Las discrepancias entre las habilidades cognoscitivas y el rendimiento deben ser vistas con cautela debido a que las DA pueden existir sin la presencia de una discrepancia numérica. No se deben pasar por alto factores del desarrollo incluyendo la edad, y la experiencia académica al valorar tales discrepancias.

D. Debe evitarse la sobreidentificación que puede presentarse cuando se consideran factores específicos (Ver antes en Etapa 1, Literal C, c). ) que agravan las DA, sin constituir por sí mismas una de ellas. Es necesaria una formulación e interpretación cuidadosa de la información reunida con el fin de poder distinguir una DA de otros factores que resultan de "dificultades para el aprendizaje" los cuales en el presente artículo se consideran como extrínsecos, fundamento de los PAA. Dentro de dichos factores, ver la mala o inadecuada instrucción que se da al niño; condiciones fí-

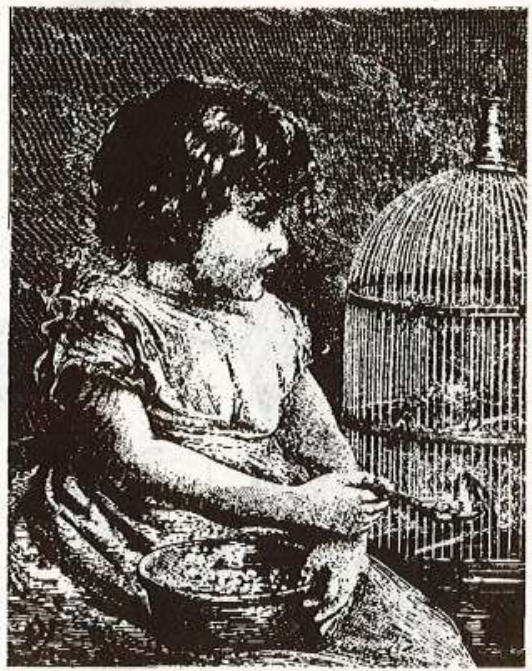

sicas, emocionales y ambientales que llevan al bajo rendimiento académico y muchas veces requieren de intervenciones complejas de la escuela y la comunidad. De no tener en cuenta lo anterior, se termina, de manera innecesaria, remitiendo al niño a programas de educación especial o a un PEP (Programa de Educación Personalizado) inadecuado.

E. Deben tenerse en cuenta los efectos de las diferencias socioculturales y del lenguaje, para descartarlas, al decidir si un PA es o no una DA.

F. En otros casos, las DA están presentes de manera simultánea con otras discapacidades o con factores extrínsecos. Brevemente, la valoración debe identificar factores específicos (tales como la pérdida unilateral e intermitente de la audición, o las dificultades de percepción social) que aunque no son responsables directos de DA, pueden ocurir concomitantemente. 


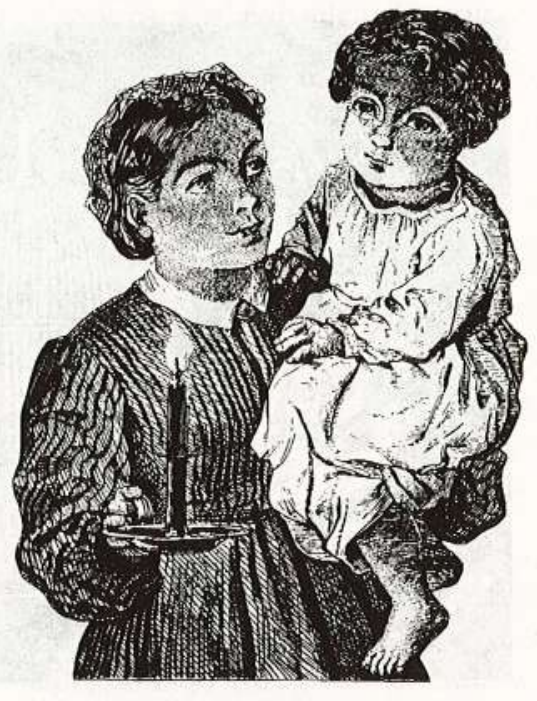

a) Individuos con otras discapacidades (retardo mental, pérdida total de la visión o de la audición,...) pueden presentar simultáneamente DA.

b) Las diferencias lingüísticas y culturales no excluyen la posibilidad de DA.

c) La instrucción inadecuada no excluye la posibilidad de DA.

\section{Decisiones}

1. Si las evidencias sustentan la presencia de una DA, debe continuarse con lo previsto en la Etapa 4 a continuación.

2. Si las evidencias no apoyan la presencia de una DA, se pueden considerar adicio- nalmente los siguientes aspectos:

a) Una discapacidad diferente a las DA, puede explicar mejor los PA del estudiante. Si esto ocurre, un estudio apropiado y técnicas de intervención para el caso deben realizarse.

b) Si se identifica alguna discapacidad $u$ otros factores extrínsecos proveen una explicación mejor del PA, deben entonces realizarse consultas adicionales al estudiante y su maestro de aula con el fin de diseñar estrategias y adaptaciones curriculares y ambientales apropiadas a las circunstancias ahora detectadas.

Conclusión: En cualquier caso, trátese de una DA o no, corresponde al equipo psicopedagógico iniciar el planeamiento y aplicación de un programa de intervención para apoyar al niño en su aprendizaje. Como ha quedado especificado, las DA, persisten durante toda la vida ${ }^{7}$, por ende, el proceso de detección de las mismas, sólo se debe realizar una vez a menos que se quiera reconfirmar el resultado. En contraste, los servicios, adaptaciones y demás estrategias que se empleen para un caso establecido de DA, pueden replantearse de acuerdo a las circuns- tancias cambiantes de la vida de la persona.

\section{Etapa 4. Elaborar programa de educación personalizada. (PEP). ${ }^{8}$}

En el Anexo 1, se describen los pasos a seguir en la elaboración de un PEP.

\section{BIBLIOGRAFÍA}

DoCKRELL, J. Y MC Shane, J. (1992) Children's Learning Difficulties. A Cognitive Approach. Oxford, UK: Blackwell Pub.

NJCLD. (1997) Operationalizing the NJCLD Definition of Learning Disabilities for Ongoing Assessment in Schools. Autor. Internet: http.//www. idonline.org/njcld/operationalizing. html-

Reis, Neu y Mcguire. (1997) Case Studies of High Ability Students with Learning Disabilities Who Have Achieved. Exceptional Children. Vol. $63,463-479$.

7 Reis, Neu y Mcguire. (1997) Case Studies of High Ability Students with Learning Disabilities Who Have Achieved. Exceptional Children. Vol. 63, 463-479.

- Ver Anexo 1. ARIAS, Juan de Dios. Elaboración de un Programa de Educación Personalizada.(PEP). Estudios en Pedagogía y Didáctica. Vol. 1. No. 2. 1996. Santafé de Bogotá. Pág. 33-40. 